\title{
Scattering from charged black holes and supergravity
}

\author{
Luís C. B. Crispino, ${ }^{1, *}$ Sam R. Dolan, ${ }^{2, \dagger}$ Atsushi Higuchi, ${ }^{3, \ddagger}$ and Ednilton S. de Oliveira ${ }^{1, \S}$ \\ ${ }^{1}$ Faculdade de Física, Universidade Federal do Pará, 66075-110, Belém, Pará, Brazil \\ ${ }^{2}$ Consortium for Fundamental Physics, School of Mathematics and Statistics, University of Sheffield, \\ Hicks Building, Hounsfield Road, Sheffield S3 7RH, United Kingdom \\ ${ }^{3}$ Department of Mathematics, University of York, Heslington, York YO10 5DD, United Kingdom
}

(Received 15 July 2015; published 26 October 2015)

\begin{abstract}
The scattering cross sections of the unpolarized electromagnetic and gravitational waves are shown to be equal for the extreme Reissner-Nordström black hole using $N=2$ supergravity. The conversion cross sections between the unpolarized electromagnetic and gravitational waves are also shown to coincide. The gravitational and electromagnetic scattering cross sections are computed numerically for ReissnerNordström black holes for several charge-to-mass ratios and the coincidence between the two scattering cross sections for the extremal case is confirmed.
\end{abstract}

DOI: 10.1103/PhysRevD.92.084056

PACS numbers: 04.40.-b, 04.70.-s, 11.80.-m

\section{INTRODUCTION}

At the center of our galaxy, an intense compact radio source, Sagittarius $\mathrm{A}^{*}$, is associated with a supermassive black hole of mass $M \sim 4.31 \times 10^{6} M_{\odot}$ [1]. It is thought that most galaxies have a supermassive core, with a close link between the properties of the black hole and the galaxy itself. Each galaxy also plays host to myriad black holes formed by stellar collapse. Clearly, black holes are a fundamental constituent of the universe, whose exotic properties are still being understood.

According to the no-hair conjecture, when a black hole in electrovacuum is perturbed, it settles back into a Kerr-Newman configuration characterized by just three numbers: mass $M$, angular momentum $J$ and electric charge $Q$ [2]. Observations suggest astrophysical black holes have significant angular momentum [3]; it is not known whether they are also endowed with charge though a Schwinger process would rapidly decrease the charge unless $M \gtrsim 10^{5} M_{\odot}$ [4]. In the extremal limit $(J / M)^{2}+$ $Q^{2} \rightarrow M^{2}$ (with natural units $G=c=1$ ), the surface gravity of the horizon, and thus the Hawking temperature, approaches zero. Extremal black holes may be regarded as solitons of Einstein-Maxwell theory [5].

In this paper we consider planar electromagnetic and gravitational waves interacting with Reissner-Nordström (RN) black holes, with $J=0,0 \leq Q \leq M$. A wave impinging upon a black hole will be partially absorbed and scattered, and the polarized flux will bear the imprint of the black hole. For example, strong-field scattering near the black hole light-ring generates a distinctive "spiral scattering" interference, with a "glory" on-axis [6]. When the

\footnotetext{
*rispino@ufpa.br

†s.dolan@sheffield.ac.uk

atsushi.higuchi@york.ac.uk

§ednilton@pq.cnpq.br
}

black hole possesses charge, the electromagnetic and gravitational perturbations are mixed together: an incident electromagnetic wave will generate a gravitational wave, and vice versa [7]. Furthermore, the helicity of the incident wave is not conserved, leading to novel signatures [8].

Numerical investigations have revealed curious coincidences between electromagnetic and gravitational scattering properties in the extremal limit $Q \rightarrow M$. First, the resonant (i.e. quasinormal mode) frequencies of gravitational waves with a multipole index $\ell$ coincide with those of electromagnetic waves with a multipole index $\ell-1$ [9]. Second, the absorption cross sections for gravitational and electromagnetic waves are equal [10]. Third, as shown here for the first time, the unpolarized (or circularly polarized) electromagnetic and gravitational scattering cross sections are equal (see Fig. 1, lower panel), and the electromagneticgravitational conversion cross sections are also equal.

These properties all hint at an underlying symmetry. Remarkably, the symmetry may be revealed through the methods of $N=2$ supergravity: a (partial) realization of Einstein's dream of unifying electromagnetism and gravity [11]. Supergravity, as the gauge theory of supersymmetry, pairs together bosonic and fermionic fields. In the $N=2$ model, the electromagnetic $(s=1)$ and gravitational $(s=2)$ fields are joined by gravitino fields $(s=3 / 2)$. The extremal RN black hole is a Bogomol'nyi-PrasadSommerfeld (BPS) state in $N=2$ supergravity, and thus it possesses an unbroken supersymmetry [5]. This supersymmetry has previously been shown to be responsible for the equality in quasinormal frequencies [12]. It also leads to some relations among partial-wave scattering amplitudes [13]. These relations have been used to explain the equality in the gravitational and electromagnetic absorption cross sections mentioned above. Here, with a novel analysis that applies supersymmetry transformations directly to planewave solutions, we show that supersymmetry accounts for all the additional coincidences noted above. 

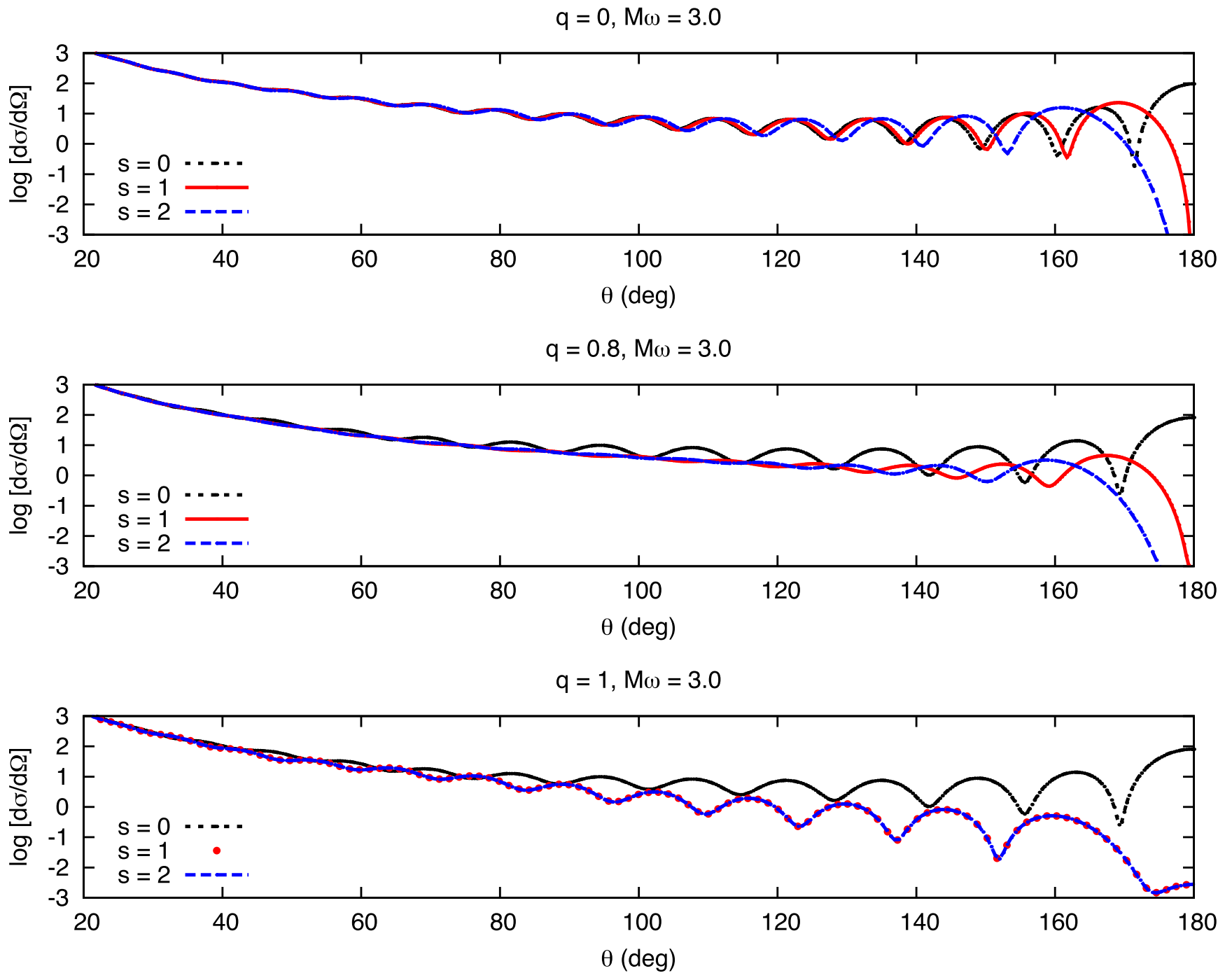

FIG. 1 (color online). Scattering cross section for scalar $(s=0)$, electromagnetic $(s=1)$, and gravitational ( $s=2)$ radiation for Schwarzschild black holes (top), Reissner-Nordström with $q \equiv|Q| / M=0.8$ (middle), and extreme ReissnerNordström black holes (bottom). Here, $M \omega=3.0$.

\section{ANALYSIS}

Majumdar-Papapetrou geometries take the form [14]

$$
d s^{2}=u^{-2} d t^{2}-u^{2}\left(d x^{2}+d y^{2}+d z^{2}\right),
$$

where $d s^{2}=g_{\mu \nu} d x^{\mu} d x^{\nu}, A_{\mu}=\left(u^{-1}, 0,0,0\right)$, and we adopt the signature $(+,-,-,-)$. With this ansatz, the EinsteinMaxwell equations reduce to Laplace's equation $\nabla^{2} u=0$ and $\partial u / \partial t=0$ on a flat background. The fundamental solution, $u=1+M / \rho$ with $\rho^{2}=x^{2}+y^{2}+z^{2}$, is recognized as the extremal $\mathrm{RN}$ spacetime in isotropic coordinates.

In $N=2$ supergravity, the gravitational and electromagnetic fields are paired with gravitino fields represented by two Majorana spinors, $\psi_{\mu}^{(I)}(I=1,2)$. The Lagrangian
$\mathcal{L}_{\text {SuGra }}\left(g_{\mu \nu}, A_{\mu}, \psi_{\mu}^{(I)}\right)$ reduces to the standard EinsteinMaxwell Lagrangian $\mathcal{L}_{\mathrm{EM}}\left(g_{\mu \nu}, A_{\mu}\right)$ when $\psi_{\mu}^{(I)}=0$. Thus, RN black holes are solutions in $N=2$ supergravity.

The Lagrangian $\mathcal{L}_{\text {SuGra }}$ is invariant, up to a total divergence, under certain gauge transformations that mix $g_{\mu \nu}, A_{\mu}$ and $\psi_{\mu}^{(I)}(N=2$ local supersymmetry). Since the extreme RN spacetime preserves some of the supersymmetry, one may generate electrovacuum solutions that are small perturbations of this spacetime from $N=2$ supergravity solutions with small gravitino perturbations by applying supersymmetry transformations.

The local supersymmetry transformation for the suitably normalized metric perturbation $h_{\mu \nu}$ and the electromagnetic field $A_{\mu}$ can be given as follows, up to a common constant factor [11]: 


$$
\begin{gathered}
\delta A_{\mu}=\epsilon^{(1) T} \gamma^{0} \psi_{\mu}^{(2)}-\epsilon^{(2) T} \gamma^{0} \psi_{\mu}^{(1)} \\
\delta h_{\mu \nu}=\frac{1}{\sqrt{2}}\left(\epsilon^{(1) T} \gamma^{0} \gamma_{(\mu} \psi_{\nu)}^{(1)}+\epsilon^{(2) T} \gamma^{0} \gamma_{(\nu} \psi_{\mu)}^{(2)}\right),
\end{gathered}
$$

where $\epsilon^{(I)}(I=1,2)$ are Majorana spinors and ${ }^{T}$ denotes the transpose. The $\gamma$-matrices satisfy $\left\{\gamma^{A}, \gamma^{B}\right\} \equiv$ $\gamma^{A} \gamma^{B}+\gamma^{B} \gamma^{A}=-2 \eta^{A B}$, where $\eta^{A B}$ is the flat Lorentzian metric. We choose the $\gamma$-matrices to be real, noting that $\gamma^{0}$ is then antisymmetric whereas $\gamma^{A}(A=1,2,3)$ are symmetric. We define $\gamma_{\mu}=\gamma^{A} e_{A \mu}$, where the vectors $e_{A}^{\mu}(A=0,1,2,3)$ form a local Lorentz frame at each spacetime point. The supersymmetry transformation of the gravitino fields can be given in terms of the field $\psi_{\mu}:=\psi_{\mu}^{(1)}+i \psi_{\mu}^{(2)}$ and the gauge function $\epsilon:=\epsilon^{(1)}+i \epsilon^{(2)}$ as follows [5] with $F_{\mu \nu}=$ $\partial_{\mu} A_{\nu}-\partial_{\nu} A_{\mu}$ on a background solution with $\psi_{\mu}^{(I)}=0$ :

$$
\delta \psi_{\mu}=D_{\mu} \epsilon+\frac{i}{4} F_{\alpha \beta} \gamma^{\alpha} \gamma^{\beta} \gamma_{\mu} \epsilon
$$

where $D_{\mu}$ is the covariant derivative for spinors.

In Majumdar-Papapetrou spacetimes there are Killing spinors $\epsilon$ satisfying $\delta \psi_{\mu}=0$ in Eq. (4). Any spinor $\epsilon=u^{-1 / 2} \epsilon_{c}$, where $\epsilon_{c}$ is constant, is a Killing spinor if $i \gamma^{0} \epsilon_{c}=-\epsilon_{c}$. This condition can be written in the real form as $\epsilon^{(2)}=-\gamma^{0} \epsilon^{(1)}$. Thus, the spinor $\epsilon^{(1)}$ can be chosen so that it is any real constant spinor at spatial infinity.

The existence of a Killing spinor implies that $N=2$ supergravity theory linearized about the extreme RN spacetime is supersymmetric. Therefore, we can map gravitino scattering solutions to photon-graviton scattering solutions by supersymmetry. More specifically, if $\psi_{\mu}^{(I)}$ give a gravitino scattering solution, then from Eqs. (2) and (3) and the relation $\epsilon^{(2)}=-\gamma^{0} \epsilon^{(1)}$ we find that, if $\epsilon=\epsilon^{(1)}+$ $i \epsilon^{(2)}$ is a Killing spinor, then the following fields will give a photon-graviton scattering solution:

$$
\begin{gathered}
A_{\mu}=\epsilon^{(1) T}\left(\psi_{\mu}^{(1)}+\gamma^{0} \psi_{\mu}^{(2)}\right), \\
h_{\mu \nu}=\frac{1}{\sqrt{2}}\left(\epsilon^{(1) T} \gamma^{0} \gamma_{(\mu} \psi_{\nu)}^{(1)}-\epsilon^{(1) T} \gamma_{(\mu} \psi_{\nu)}^{(2)}\right) .
\end{gathered}
$$

Since we are only interested in linearized field equations, we can treat $\psi_{\mu}^{(I)}$ and $\epsilon^{(I)}$ as ordinary functions of spacetime rather than fermionic operators or Grassmann numbers.

In order to discuss gravitino scattering solutions we first should consider gravitino plane-wave solutions in flat spacetime. The noninteracting gravitino fields $\psi_{\mu}^{(I)}$ satisfy $\gamma_{[\mu} \partial_{\nu} \psi_{\lambda]}^{(I)}=0$ in flat space [15]. These equations are invariant under the following gauge transformations: $\psi_{\mu}^{(I)} \mapsto \psi_{\mu}^{(I)}+\partial_{\mu} \eta^{(I)}$, where $\eta^{(I)}$ are any Majorana spinors. Let us consider a gravitino plane wave incident from $z=\infty$. Under the gauge condition $\gamma^{\mu} \psi_{\mu}^{(I)}=0$ the general solutions to these field equations are indeed plane waves and take the form $\psi_{\mu}^{(I)}=\psi_{\mu}^{(I, c)} e^{-i k(t+z)}$, where $\psi_{\mu}^{(I, c)}$ are constant spinor-vectors. The gauge invariance mentioned above can be used to gauge away the components $\psi_{0}^{(I)}$ and $\psi_{3}^{(I)}$ (see, e.g., Refs. [11,16]). Then we find $\gamma^{0} \gamma^{3} \psi_{\mu}^{(I)}=\psi_{\mu}^{(I)}$, $\mu=1,2$ and $\psi_{2}^{(I)}=\gamma^{1} \gamma^{2} \psi_{1}^{(I)}$. From now on we let $\psi_{\mu}^{(I)}$ be a scattering solution in the extreme RN background which becomes a plane wave as described above in the limit $z \rightarrow \infty$.

For $z \rightarrow \infty$ the components with $\mu=1$ and 2 of the electromagnetic plane wave in Eq. (5) may be nonzero and are given by

$$
\begin{gathered}
A_{1} \approx \epsilon^{(1) T}\left(\psi_{1}^{(1)}+\gamma^{0} \psi_{1}^{(2)}\right), \\
A_{2} \approx \epsilon^{(1) T} \gamma^{1} \gamma^{2}\left(\psi_{1}^{(1)}+\gamma^{0} \psi_{1}^{(2)}\right),
\end{gathered}
$$

where we have used the relation $\psi_{2}^{(I)} \approx \gamma^{1} \gamma^{2} \psi_{1}^{(I)}$. We also find that, for $z \rightarrow \infty$, the $h_{\mu \nu}$ given by Eq. (6) is nonzero only if $\mu$ or $\nu$ is either 1 or 2 . It can also be shown that the $h_{\mu 0}$ and $h_{\mu 3}$ are of pure-gauge form, and hence can be disregarded in considering the flux of gravitational wave coming in from $z=\infty$. Hence, the only relevant components of the gravitational plane wave described by Eq. (6) are found for large $z$ as

$$
\begin{gathered}
h_{22} \approx-h_{11} \approx \frac{1}{\sqrt{2}} \epsilon^{(1) T} \gamma^{0} \gamma^{1}\left(\psi_{1}^{(1)}-\gamma^{0} \psi_{1}^{(2)}\right), \\
h_{12} \approx-\frac{1}{\sqrt{2}} \epsilon^{(1) T} \gamma^{0} \gamma^{2}\left(\psi_{1}^{(1)}-\gamma^{0} \psi_{1}^{(2)}\right) .
\end{gathered}
$$

We note that these expressions and Eqs. (7) and (8) become exact in the limit $z \rightarrow \infty$, where $\psi_{\mu}^{(I)}$ gives a plane wave.

Now, we shall find gravitino plane-wave solutions that give linearly polarized incident electromagnetic plane waves through Eq. (5) but no incident gravitational plane waves through Eq. (6). We first note that the 4-dimensional space of constant real spinors consists of four 1-dimensional eigenspaces of the commuting real symmetric matrices $i \gamma_{5} \gamma^{3}$ and $\gamma^{2}$, each with eigenvalues \pm 1 , where $\gamma_{5}:=-i \gamma^{0} \gamma^{1} \gamma^{2} \gamma^{3}$. We choose the scattering solution $\psi_{\mu}^{(I)}$ such that $\psi_{1}^{(1)}+\gamma^{0} \psi_{1}^{(2)}$ is an eigenspinor of $i \gamma_{5} \gamma^{3}$ and $\gamma^{2}$ with eigenvalues +1 in the limit $z \rightarrow \infty$ where $\psi_{\mu}^{(I)}$ is an incoming plane wave. Since $\psi_{1}^{(I)} \approx \gamma^{0} \gamma^{3} \psi_{1}^{(I)}$ for $z \rightarrow \infty$, we have $\psi_{1}^{(1)}-\gamma^{0} \psi_{1}^{(2)} \approx \gamma^{0} \gamma^{3}\left(\psi_{1}^{(1)}+\gamma^{0} \psi_{1}^{(2)}\right)$. Thus, $\psi_{1}^{(1)}-$ $\gamma^{0} \psi_{1}^{(2)}$ is an eigenspinor of $i \gamma_{5} \gamma^{3}$ with eigenvalue -1 for large $z$. Define $\epsilon_{+ \pm}$to be unit real spinors satisfying $i \gamma_{5} \gamma^{3} \epsilon_{+ \pm}=\epsilon_{+ \pm}$and $\gamma^{2} \epsilon_{+ \pm}= \pm \epsilon_{+ \pm}$. Then, we find from Eqs. (7)-(8) and Eqs. (9)-(10) that the incident 
electromagnetic wave is linearly polarized in the $1 \mathrm{st}$ direction if $\epsilon^{(1)}=\epsilon_{++}$and in the 2nd direction if $\epsilon^{(1)}=$ $\epsilon_{+-}$. In either case there will be no incident gravitational wave.

Let us now discuss the differential scattering and conversion cross sections for unpolarized incident electromagnetic waves [17]. Far away from the origin a scattered solution can locally be approximated by a plane wave times $1 / r$. Now, choose a rotated coordinate system characterized by the orthonormal vectors $\mathbf{e}_{\hat{i}}, \hat{i}=\hat{1}, \hat{2}, \hat{3}$, such that the unit vector in the 3 rd direction in the old coordinate system, $\mathbf{e}_{3}$, is expressed as $\mathbf{e}_{3}=n_{\hat{1}} \mathbf{e}_{\hat{1}}+n_{\hat{2}} \mathbf{e}_{\hat{2}}+n_{\hat{3}} \mathbf{e}_{\hat{3}}$. After a suitable gauge transformation, the electromagnetic and gravitational plane waves in Eqs. (5) and (6), respectively, propagating in the negative $\hat{3}$-direction are given by replacing the coordinates 1, 2 and 3 by $\hat{1}, \hat{2}$ and $\hat{3}$ in Eqs. (7)-(8) and Eqs. (9)(10). Hence the differential scattering cross section in the negative $\hat{3}$-direction is

$$
\begin{aligned}
\left.\frac{d \sigma}{d \Omega}\right|_{A \rightarrow A}= & C \sum_{ \pm} \lim _{r \rightarrow \infty} r^{2}\left(\left|A_{\hat{1}}\right|^{2}+\left|A_{\hat{2}}\right|^{2}\right) \\
= & C \lim _{r \rightarrow \infty} r^{2}\left(\psi_{\hat{1}}^{(1) \dagger}-\psi_{\hat{1}}^{(2) \dagger} \gamma^{0}\right) \\
& \times\left(\Sigma_{+}+\gamma^{\hat{2}} \gamma^{\hat{1}} \Sigma_{+} \gamma^{\hat{1}} \gamma^{\hat{2}}\right)\left(\psi_{\hat{1}}^{(1)}+\gamma^{0} \psi_{\hat{1}}^{(2)}\right),
\end{aligned}
$$

where $C$ is a constant and $\Sigma_{+}=\epsilon_{++} \epsilon_{++}^{T}+$ $\epsilon_{+-} \epsilon_{+-}^{T} \approx\left(1+i \gamma_{5} \gamma^{3}\right) / 2$. By substituting this formula into Eq. (11) and using $\gamma^{3}=n_{\hat{1}} \gamma^{\hat{1}}+n_{\hat{2}} \gamma^{\hat{2}}+n_{\hat{3}} \gamma^{\hat{3}}$, we find

$$
\begin{aligned}
\left.\frac{d \sigma}{d \Omega}\right|_{A \rightarrow A}= & C \lim _{r \rightarrow \infty} r^{2}\left(\psi_{\hat{1}}^{(1) \dagger}-\psi_{\hat{1}}^{(2) \dagger} \gamma^{0}\right) \\
& \times\left(1+i n_{\hat{3}} \gamma_{5} \gamma^{\hat{3}}\right)\left(\psi_{\hat{1}}^{(1)}+\gamma^{0} \psi_{\hat{1}}^{(2)}\right) .
\end{aligned}
$$

The matrices $\gamma^{0}$ and $\gamma^{0} \gamma^{\hat{3}}$ anticommute. From this and the fact that $\psi_{\hat{1}}^{(I)}$ in the large- $r$ limit in the negative $\hat{3}$-direction is an eigenspinor of $\gamma^{0} \gamma^{\hat{3}}$ with eigenvalue +1 , we conclude that $\gamma^{0} \psi_{\hat{1}}^{(I)}$ is an eigenspinor of $\gamma^{0} \gamma^{\hat{3}}$ with eigenvalue -1 in this limit. Hence $\psi_{\hat{1}}^{(I)}$ and $\gamma^{0} \psi_{\hat{1}}^{(J)}$ become orthogonal in this limit. Therefore,

$$
\begin{gathered}
\lim _{r \rightarrow \infty} r^{2}\left(\psi_{\hat{1}}^{(1) \dagger}-\psi_{\hat{1}}^{(2) \dagger} \gamma^{0}\right)\left(\psi_{\hat{1}}^{(1)}+\gamma^{0} \psi_{\hat{1}}^{(2)}\right) \\
=\lim _{r \rightarrow \infty} r^{2}\left(\psi_{\hat{1}}^{(1) \dagger} \psi_{\hat{1}}^{(1)}+\psi_{\hat{1}}^{(2) \dagger} \psi_{\hat{1}}^{(2)}\right) .
\end{gathered}
$$

Similarly, since $\gamma_{5} \gamma^{\hat{3}}$ anticommutes with $\gamma^{0} \gamma^{\hat{3}}$, we find that $\psi_{\hat{1}}^{(I)}$ and $\gamma_{5} \gamma^{\hat{3}} \psi_{\hat{1}}^{(J)}$ become orthogonal in this limit. Hence

$$
\begin{gathered}
\lim _{r \rightarrow \infty} r^{2}\left(\psi_{\hat{1}}^{(1) \dagger}-\psi_{\hat{1}}^{(2) \dagger} \gamma^{0}\right) \gamma_{5} \gamma^{\hat{3}}\left(\psi_{\hat{1}}^{(1) \dagger}+\gamma^{0} \psi_{\hat{1}}^{(2) \dagger}\right) \\
=\lim _{r \rightarrow \infty} r^{2}\left(\psi_{\hat{1}}^{(2) \dagger} \gamma_{5} \psi_{\hat{1}}^{(1)}-\psi_{\hat{1}}^{(1) \dagger} \gamma_{5} \psi_{\hat{1}}^{(2)}\right),
\end{gathered}
$$

where we have used the fact that $\psi_{\hat{1}}^{(I)}$ become eigenspinors of $\gamma^{0} \gamma^{\hat{3}}$ with eigenvalue +1 in this limit. By substituting Eqs. (13) and (14) into Eq. (12) we obtain

$$
\left.\frac{d \sigma}{d \Omega}\right|_{A \rightarrow A}=\mathcal{A}+n_{\hat{3}} \mathcal{B},
$$

where

$$
\begin{gathered}
\mathcal{A}:=C \lim _{r \rightarrow \infty} r^{2}\left(\psi_{\hat{1}}^{(1) \dagger} \psi_{\hat{1}}^{(1)}+\psi_{\hat{1}}^{(2) \dagger} \psi_{\hat{1}}^{(2)}\right), \\
\mathcal{B}:=i C \lim _{r \rightarrow \infty} r^{2}\left(\psi_{\hat{1}}^{(2) \dagger} \gamma_{5} \psi_{\hat{1}}^{(1)}-\psi_{\hat{1}}^{(1) \dagger} \gamma_{5} \psi_{\hat{1}}^{(2)}\right) .
\end{gathered}
$$

The differential cross section in the same direction for the conversion from (unpolarized) electromagnetic wave to gravitational wave can be found similarly, using Eqs. (9) and (10), as

$$
\begin{aligned}
\left.\frac{d \sigma}{d \Omega}\right|_{A \rightarrow h}= & C \sum_{ \pm} \lim _{r \rightarrow \infty} r^{2}\left(\left|h_{\hat{1} \hat{1}}\right|^{2}+\left|h_{\hat{2} \hat{2}}\right|^{2}+2\left|h_{\hat{1} \hat{2}}\right|^{2}\right) \\
= & C \lim _{r \rightarrow \infty} r^{2}\left(\psi_{\hat{1}}^{(1) \dagger}+\psi_{\hat{1}}^{(2) \dagger} \gamma^{0}\right) \\
& \times\left(1+i n_{\hat{3}} \gamma_{5} \gamma^{\hat{3}}\right)\left(\psi_{\hat{1}}^{(1)}-\gamma^{0} \psi_{\hat{1}}^{(2)}\right) \\
= & \mathcal{A}-n_{\hat{3}} \mathcal{B} .
\end{aligned}
$$

Next, we let $\epsilon^{(1)}=\epsilon_{- \pm}$, where $\epsilon_{- \pm}$are real unit spinors satisfying $i \gamma_{5} \gamma^{3} \epsilon_{- \pm}=-\epsilon_{- \pm}$and $\gamma^{2} \epsilon_{- \pm}= \pm \epsilon_{- \pm}$. Then, we find from Eqs. (7)-(8) and Eqs. (9)-(10) that the solutions given by Eqs. (5) and (6) are such that there is a linearlypolarized incident gravitational wave with the same flux as above coming in from $z=\infty$ with the " + " polarization (the " $x$ " polarization) if $\epsilon^{(1)}=\epsilon_{-+}\left(\epsilon^{(1)}=\epsilon_{--}\right)$. In either case there will be no incident electromagnetic wave. We can express the unpolarized differential scattering cross section in the negative $\hat{3}$-direction as:

$$
\begin{aligned}
\left.\frac{d \sigma}{d \Omega}\right|_{h \rightarrow h} & =C \sum_{ \pm} \lim _{r \rightarrow \infty} r^{2}\left(\left|h_{\hat{1} \hat{1}}\right|^{2}+\left|h_{\hat{2} \hat{2}}\right|^{2}+2\left|h_{\hat{1} \hat{2}}\right|^{2}\right) \\
& =\mathcal{A}+n_{\hat{3}} \mathcal{B},
\end{aligned}
$$

where the summation is over $\epsilon_{-+}$. We have used $\epsilon_{-+} \epsilon_{-+}^{T}+\epsilon_{--} \epsilon_{--}^{T} \approx\left(1-i \gamma_{5} \gamma^{3}\right) / 2$. We similarly find the differential conversion cross section from gravitational to electromagnetic waves in the negative $\hat{3}$-direction as 
$\left.\frac{d \sigma}{d \Omega}\right|_{h \rightarrow A}=C \sum_{ \pm} \lim _{r \rightarrow \infty} r^{2}\left(\left|A_{\hat{1}}\right|^{2}+\left|A_{\hat{2}}\right|^{2}\right)=\mathcal{A}-n_{\hat{3}} \mathcal{B}$.

By comparing Eqs. (15) and (19) we see that the scattering cross sections for the gravitational and electromagnetic waves are equal. We also find, by comparing Eqs. (18) and (20), that the conversion cross sections from electromagnetic to gravitational waves and from gravitational to electromagnetic waves are equal.

\section{NUMERICAL RESULTS}

We now show that equality of scattering cross sections is supported by new numerical results, obtained via a rather different formalism: partial-wave solutions constructed from multipolar sums. For this purpose, we compute the scattering cross section of incident gravitational plane waves by RN black holes and compare it with the electromagnetic counterpart [8].

The multipoles of the electromagnetic and gravitational perturbations are given by [18]:

$$
\begin{aligned}
F_{\ell}^{\mathcal{P}} & =\varphi_{\ell+}^{\mathcal{P}} \cos \psi-\varphi_{\ell-}^{\mathcal{P}} \sin \psi, \\
G_{\ell}^{\mathcal{P}} & =\varphi_{\ell+}^{\mathcal{P}} \sin \psi+\varphi_{\ell-}^{\mathcal{P}} \cos \psi,
\end{aligned}
$$

where the functions $\varphi_{\ell \pm}^{\mathcal{P}}$ obey the equations

$$
\frac{d^{2}}{d r_{*}^{2}} \varphi_{\ell \pm}^{\mathcal{P}}+\left(\omega^{2}-V_{\ell \pm}^{\mathcal{P}}\right) \varphi_{\ell \pm}^{\mathcal{P}}=0 .
$$

Here, $\mathcal{P}= \pm$ denotes parity (even [polar] or odd [axial]), $r_{*}$ is the tortoise coordinate, given by $d / d r_{*} \equiv f(r) d / d r$ with $f(r)=1-2 M / r+Q^{2} / r^{2}$ in the standard coordinate system, and

$$
\sin (2 \psi)=-2 \mathcal{P} Q \frac{[(\ell-1)(\ell+2)]^{1 / 2}}{\Omega}, \quad|\psi|<\frac{\pi}{4},
$$

with $\Omega=\sqrt{9 M^{2}+4 Q^{2}(\ell-1)(\ell+2)}$.

The effective potentials, $V_{\ell \pm}^{\mathcal{P}}$, can be found, for example, in Ref. [19], with plots in Ref. [20]. As a general behavior, $V_{\ell \pm}^{\mathcal{P}}$ are zero at the event horizon, $r_{+}$, and at infinity. Therefore, it is possible to write:

$$
\varphi_{\ell \pm}^{\mathcal{P}}\left(r_{*}\right) \sim\left\{\begin{array}{lc}
e^{-i \omega r_{*}}+A_{ \pm, \omega \ell}^{\mathcal{P}} e^{i \omega r_{*}}, & \left(r_{*} \rightarrow \infty\right) ; \\
B_{ \pm, \omega \ell}^{\mathcal{P}} e^{-i \omega r_{*}}, & \left(r_{*} \rightarrow-\infty\right) .
\end{array}\right.
$$

The gravitational scattering cross section for RN black holes can be written as:

$$
\begin{gathered}
\left.\frac{d \sigma}{d \Omega}\right|_{h \rightarrow h}=|\mathcal{F}|^{2}+|\mathcal{G}|^{2}, \\
\mathcal{F}=\frac{\pi}{i \omega} \sum_{\mathcal{P}= \pm 1} \sum_{\ell=2}^{\infty} Z_{\ell}(1) Z_{\ell}(\cos \theta)\left[e^{2 i \delta_{\ell}^{\mathcal{P}}(\omega)}-1\right],
\end{gathered}
$$

$\mathcal{G}=\frac{\pi}{i \omega} \sum_{\mathcal{P}= \pm 1} \sum_{\ell=2}^{\infty} \mathcal{P}(-1)^{\ell} Z_{\ell}(1) Z_{\ell}(-\cos \theta)\left[e^{2 i \delta_{\ell}^{\mathcal{P}}(\omega)}-1\right]$,

where $Z_{\ell} \equiv_{s=-2} Y_{\ell}^{m=2}(\cdot)$ are the spin-weighted spherical harmonics [21], and the phase shifts are given by

$$
e^{2 i \delta_{\ell}^{\mathcal{P}}(\omega)}=(-1)^{\ell+1}\left(A_{-, \omega \ell}^{\mathcal{P}} \cos ^{2} \psi+A_{+, \omega \ell}^{\mathcal{P}} \sin ^{2} \psi\right) .
$$

The $\mathcal{F}$ and $\mathcal{G}$ are the helicity-preserving and the helicityreversing amplitudes, respectively. We note that $\mathcal{G}$ is associated with the difference of phase shifts from distinct parities and, therefore, is zero whenever $\delta_{\ell}^{+}(\omega)=\delta_{\ell}^{-}(\omega)$. This is the case, e.g., for electromagnetic radiation around a Schwarzschild $(Q=0)$ black hole, but not for a ReissnerNordström black hole. Observational consequences of this "helicity-nonconservation" were explored in Ref. [8].

Figure 1 shows numerically computed scattering cross sections for waves of spin 0,1 , and 2 for black holes with $q=Q / M=0,0.8,1$ and $M \omega=3.0$. The cross sections for Schwarzschild black holes are broadly similar across the range $\theta \lesssim 100^{\circ}$, but differ at large angles. For $q=0.8$, results for $s=1,2$ are very close for $\theta \lesssim 100^{\circ}$, but differ significantly from the scalar case. For extremal RN black holes $(q=1)$, we see excellent numerical confirmation that the electromagnetic and gravitational scattering cross sections are equal (lower panel).

\section{SUMMARY AND DISCUSSION}

In this paper we studied the scattering properties of the unpolarized gravitational and electromagnetic waves from the Reissner-Nordström black hole. In particular, we showed that the differential scattering cross sections for these two bosonic fields become equal when the black hole is extremal, and we verified this result using the partialwave approach with a numerical method. We also showed that the conversion cross sections between the electromagnetic and gravitational waves coincide in the extremal limit.

To expose the symmetry that underlies such coincidences, we used $N=2$ supergravity to map the gravitino scattering solutions to the electromagnetic and gravitational scattering solutions. It is clear that a similar mapping exists on any supersymmetric background spacetime with a wellposed scattering problem. Thus, it will surely be interesting to investigate similar coincidences in the scattering of bosonic fields on other supersymmetric backgrounds, using the mapping we have applied here.

\section{ACKNOWLEDGMENTS}

The authors would like to thank Gary Gibbons for useful correspondence and Conselho Nacional de Desenvolvimento Científico e Tecnológico (CNPq), 
Coordenação de Aperfeiçoamento de Pessoal de Nível Superior (CAPES), and Fundação Amazônia de Amparo a Estudos e Pesquisas do Pará (FAPESPA) for partial financial support. A. H. also acknowledges partial support from the Abdus Salam International Centre for Theoretical
Physics through the Visiting Scholar/Consultant Programme, and the Universidade Federal do Pará (UFPA) in Belém for kind hospitality. S. D. acknowledges support under STFC Grant No. ST/L000520/1 and EPSRC Grant No. EP/M025802/1.
[1] S. Gillessen, F. Eisenhauer, S. Trippe, T. Alexander, R. Genzel, F. Martins, and T. Ott, Monitoring stellar orbits around the massive black hole in the galactic center, Astrophys. J. 692, 1075 (2009).

[2] P. T. Chrusciel, J. L. Costa, and M. Heusler, Stationary black holes: Uniqueness and beyond, Living Rev. Relativity 15, 7 (2012).

[3] G. Risaliti, F. Harrison, K. Madsen, D. Walton, S. Boggs et al., A rapidly spinning supermassive black hole at the centre of NGC 1365, Nature (London) 494, 449 (2013).

[4] G. W. Gibbons, Vacuum polarization and the spontaneous loss of charge by black holes, Commun. Math. Phys. 44, 245 (1975).

[5] G. W. Gibbons and C. M. Hull, A Bogomolny bound for general relativity and solitons in $N=2$ supergravity, Phys. Lett. 109B, 190 (1982).

[6] L. C. B. Crispino, S. R. Dolan, and E. S. Oliveira, Electromagnetic Wave Scattering by Schwarzschild Black Holes, Phys. Rev. Lett. 102, 231103 (2009).

[7] D. W. Olson and W. G. Unruh, Conversion of Electromagnetic to Gravitational Radiation by Scattering from a Charged Black Hole, Phys. Rev. Lett. 33, 1116 (1974).

[8] L. C. B. Crispino, S. R. Dolan, A. Higuchi, and E. S. de Oliveira, Inferring black hole charge from backscattered electromagnetic radiation, Phys. Rev. D 90, 064027 (2014).

[9] H. Onozawa, T. Mishima, T. Okamura, and H. Ishihara, Quasinormal modes of maximally charged black holes, Phys. Rev. D 53, 7033 (1996).

[10] E. S. Oliveira, L. C. B. Crispino, and A. Higuchi, Equality between gravitational and electromagnetic absorption cross sections of extreme Reissner-Nordström black holes, Phys. Rev. D 84, 084048 (2011).

[11] P. Van Nieuwenhuizen, Supergravity, Phys. Rep. 68, 189 (1981).

[12] H. Onozawa, T. Okamura, T. Mishima, and H. Ishihara, Perturbing supersymmetric black holes, Phys. Rev. D 55, R4529 (1997).

[13] T. Okamura, Scattering off the extreme Reissner-Nordström black hole in $N=2$ supergravity, Phys. Rev. D 56, 4927 (1997).

[14] J. B. Hartle and S. W. Hawking, Solutions of the EinsteinMaxwell equations with many black holes, Commun. Math. Phys. 26, 87 (1972).

[15] W. Rarita and J. Schwinger, On a theory of particles with half-integral spin, Phys. Rev. 60, 61 (1941).

[16] G. Sterman, P. K. Townsend, and P. van Nieuwenhuizen, Unitarity, Ward identities, and new quantization rules of supergravity, Phys. Rev. D 17, 1501 (1978).

[17] Since the RN black holes are parity invariant, the cross sections for circularly polarized waves are equal to those for unpolarized waves.

[18] F. J. Zerilli, Perturbation analysis for gravitational and electromagnetic radiation in a Reissner-Nordström geometry, Phys. Rev. D 9, 860 (1974).

[19] V. Moncrief, Stability of Reissner-Nordström black holes, Phys. Rev. D 10, 1057 (1974).

[20] S. Chandrasekhar, The Mathematical Theory of Black Holes (Clarendon Press, Oxford, 1983).

[21] J. N. Goldberg, A. J. Macfarlane, E. T. Newman, F. Rohrlich, and E. C. G. Sudarshan, Spin-s Spherical Harmonics and ð, J. Math. Phys. (N.Y.) 8, 2155 (1967). 